\title{
Karp's theorem in inverse obstacle scattering problems
}

\author{
Jaemin Shin \\ Department of Mathematical Sciences \& \\ Institute of Applied Mathematics and Optics \\ Hanbat National University, Daejeon 34158, Republic of Korea \\ e-mail: jaemin.shin@hanbat.ac.kr
}

\begin{abstract}
In this work, we provide a proof of the so-called Karp's theorem in a different approach. We use the unique continuation principle together with the monotonicity of eigenvalues for the negative Laplace operator. This method is new and would be applicable to other types of inverse scattering problems.
\end{abstract}

Keywords: Inverse obstacle scattering problem, Karp's Theorem, Symmetric problem

\section{Introduction}

The inverse obstacle scattering problem deals with the determination of shapes and/or positions of objects from the knowledge of associated scattered fields, which has many applications in areas, such as medical imaging, seismic imaging, and non-destructive testing. Consider the scattered field $u^{s}$ of a plane wave $u^{i}=e^{i k x \cdot d}$ by a 2-dimensional impenetrable obstacle $\Omega$. Here, $d=(\cos \delta, \sin \delta)$ is the incident direction. Then, the total wave $u=u^{s}+u^{i}$ solves the exterior Helmholtz equation,

$$
\Delta u+k^{2} u=0 \text { in } \mathbb{R}^{2} \backslash \bar{\Omega}
$$

satisfying the so-called Sommerfeld radiation condition for $u^{s}$,

$$
\lim _{r \rightarrow \infty} \sqrt{r}\left(\frac{\partial u^{s}}{\partial r}-i k u^{s}\right)=0, \quad r:=|x| .
$$

The total field $u$, depending on the nature of the scatter, satisfies one of the following boundary conditions:

$$
\begin{aligned}
u & =0 \text { on } \partial \Omega \text { (sound-soft obstacle), } \\
\frac{\partial u}{\partial \nu} & =0 \text { on } \partial \Omega \text { (sound-hard obstacle). }
\end{aligned}
$$

Due to the radiation condition (1b), one can define the far-field pattern $u_{\infty}(\widehat{x}, d, k)$, which is a complex-valued function of receiver direction $\widehat{x}=x / r$, incident direction $d$, and wave number $k>0$ as follows.

$$
u_{\infty}(\widehat{x}, d, k)=\lim _{r \rightarrow \infty} u^{s}(x, d) \sqrt{r} e^{-i k r} .
$$

The inverse obstacle scattering problem is now formulated to determine $\partial \Omega$ from $u_{\infty}(\widehat{x}, d, k)$ defined on a certain subset $E$ of $S^{1} \times S^{1} \times \mathbb{R}_{+}^{1}$. This problem has been studied intensively for several decades analytically as well as numerically, under various a priori assumptions on the shape of the scatterer and sets $E$. We refer the reader to [1,2] and a recent review paper [3] for a comprehensive survey.

Although it is still an open problem to determine whether the uniqueness of a general scatterer from the data of $u_{\infty}(\widehat{x}, d, k)$ in $S^{1} \times\left\{d_{0}\right\} \times\left\{k_{0}\right\}$ is guaranteed, there are many uniqueness results in various aspects. One of the earliest and most interesting results is the so-called Karp's theorem [4]. Let $Q$ be a rotational matrix. If the scatterer is assumed to be a disk centered at the origin, then obviously

$$
u_{\infty}(\widehat{x}, d, k)=u_{\infty}(Q \widehat{x}, Q d, k)
$$

for all $\widehat{x}, d \in S^{1}$. Karp's theorem states the converse is also true. That is, 
Theorem 1. Let $\Omega$ be a bounded, simply connected domain with $C^{2}$ boundary $\partial \Omega$, and $Q$ be a rotation matrix. Suppose that the far-field pattern $u_{\infty}(\widehat{x}, d, k)$ satisfies (3) for a fixed $k$ and for all $\widehat{x}, d, Q$. Then, $\Omega$ is a disk centered at the origin.

Indeed, Karp showed Theorem 1 for the case of a sound-soft obstacle, (1c) in [4]. After Karp's result for the sound-soft obstacle, Colton and Kirsch extended Karp's theorem to the cases of a sound-hard scattering obstacle and scattering by an inhomogeneous medium in [5]. Additionally, similar results in electromagnetic and elastodynamic scattering problems are provided in [6,7]. It is worth to mention that Karp's theorem can be generalized to the symmetric problem for the Helmholtz equation proposed by Ramm. We refer the reader to his series of work, for instance, see $[8,9,10]$ and the references therein.

The purpose of this short note is to provide a proof of Karp's theorem using a different approach, to advance our understanding of obstacle scattering phenomena. The proof is provided in Section 2. We first construct a subdomain $D_{\varepsilon}$ of $\mathbb{R}^{2} \backslash \bar{\Omega}$ that is enclosed by a disk with radius $\varepsilon$ if the scatterer is assumed not to be a disk. Then, we show that the full-field $u$ of (1) has zero Dirichlet boundary condition on $\partial D_{\varepsilon}$ if Karp's assumption (3) is fulfilled, by the unique continuation principle. By taking $\varepsilon$ to be sufficiently small, we show $u=0$ in $D_{\varepsilon}$, which yields a contradiction. We mainly discuss the sound-soft case in two dimensions; however, this approach can be applied to the sound-hard obstacles as well as the three-dimensional case, which is briefly discussed in Section 3.

\section{Proof of Karp's theorem}

Let $Q$ be a rotation operator. We denote the solution to (1) for the rotated scatterer $Q(\Omega)$ by $v(x, d, k)=v^{s}(x, d, k)+$ $e^{i k x \cdot d}$ and the corresponding far-field pattern by $v_{\infty}(\widehat{x}, d, k)$. Then, the rotational invariance of the Laplacian gives the following relation between $u$ and $v$.

Lemma 2. Let $Q$ be a rotation operator. Then, the scattered field $u^{s}$ for (1) and $v^{s}$ for $(1)$ with $Q(\Omega)$ satisfy

$$
v^{s}(x, Q(d), k)=u^{s}\left(Q^{-1}(x), d, k\right) \quad \text { in } \mathbb{R}^{2} \backslash \overline{Q(\Omega)} .
$$

Moreover, the corresponding far-field patters $v_{\infty}$ and $u_{\infty}$ satisfy

$$
v_{\infty}(Q(\widehat{x}), Q(d), k)=u_{\infty}(\widehat{x}, d, k) .
$$

Proof. As the Laplacian is rotationally invariant, $u^{s}\left(Q^{-1}(x), d, k\right)$ solves (1a) in $\mathbb{R}^{2} \backslash \overline{Q(\Omega)}$ and satisfies (1b). Furthermore, at the boundary $\partial Q(\Omega)$

$$
\begin{aligned}
u^{s}\left(Q^{-1}(x), d, k\right) & =-e^{i k Q^{-1}(x) \cdot d} \\
& =-e^{i k x \cdot Q(d)}=v^{s}(x, Q(d), k) .
\end{aligned}
$$

As $v^{s}(x, Q(d), k)$ is the unique solution to $(1)$ for the obstacle $Q(\Omega)$, it should be identical with $u^{s}\left(Q^{-1}(x), d, k\right)$ as (4). The identity (5) follows from the integral representation of the far-field pattern (see [11]);

$$
\begin{aligned}
\sqrt{8 \pi k} e^{i \pi / 4} & v_{\infty}(Q(\widehat{x}), Q(d), k)=\int_{Q(\partial \Omega)}\left(v^{s}(y, Q(d), k) \frac{\partial}{\partial \nu(y)} e^{-i k Q(\widehat{x}) \cdot y}-e^{-i k Q \widehat{x}) \cdot y} \frac{\partial}{\partial \nu(y)} v^{s}(y, Q(d), k)\right) d s(y) \\
& =\int_{Q(\partial \Omega)}\left(u^{s}\left(Q^{-1}(y), d, k\right) \frac{\partial}{\partial \nu(y)} e^{-i k \widehat{x} \cdot Q^{-1}(y)}-e^{-i k \widehat{x} \cdot Q^{-1}(y)} \frac{\partial}{\partial \nu(y)} u^{s}\left(Q^{-1}(y), d, k\right)\right) d s(y) \\
& =\int_{\partial \Omega}\left(u^{s}(z, d, k) \frac{\partial}{\partial \nu(z)} e^{-i \hat{x} \cdot z}-e^{-i \widehat{x} \cdot z} \frac{\partial}{\partial \nu(z)} u^{s}(z, d, k)\right) d s(z) \\
& =\sqrt{8 \pi k} e^{i \pi / 4} u_{\infty}(\widehat{x}, d, k) .
\end{aligned}
$$

We remark that (4) does not imply

$$
v^{s}(Q(x), Q(d), k)=u^{s}(x, d, k)
$$

because the domains of $v^{s}(Q(x), Q(d), k)$ and $u^{s}(x, d, k)$ are different. However, (6) holds in $\mathbb{R}^{2} \backslash \overline{B(0, R)}$ for a sufficiently large $R$, which yields (5).

The following is the well-known Rellich's Lemma. A proof can be found in most textbooks, e.g., [1, 11]. 
Lemma 3. Suppose that $u_{i, \infty}$ is a far-field pattern of (1) for fixed $d_{i}, k_{i}, \Omega_{i}(i=1,2)$ such that

$$
u_{1, \infty}\left(\widehat{x}, d_{1}, k_{1}, \Omega_{1}\right)=u_{2, \infty}\left(\widehat{x}, d_{2}, k_{2}, \Omega_{2}\right)
$$

Then,

where $B \supset \Omega_{1} \cup \Omega_{2}$.

$$
u_{1}^{s}\left(x, d_{1}, k_{1}, \Omega_{1}\right)=u_{2}^{s}\left(x, d_{2}, k_{2}, \Omega_{2}\right) \quad \text { in } \mathbb{R}^{2} \backslash \bar{B}
$$

Now, we show Theorem 1 using a contradiction argument based on the unique continuation principle. We assume that the obstacle $\Omega$ is not a disk centered at the origin. Then, for a given $\varepsilon>0$, we may construct a subdomain $D_{\varepsilon}$ of $\mathbb{R}^{2} \backslash \bar{\Omega}$, which is contained in a ball with radius $\varepsilon$.

To this end, we consider the smallest open ball $B:=B(0, R)=\left\{x \in \mathbb{R}^{2}:|x|<R\right\}$ containing $\Omega$. Then, trivially, $\partial \Omega \cap \partial B$ is not the empty set. Let $\Gamma$ be a connected component of $\partial \Omega \cap \partial B$, and $x_{0}, x_{1}$ be the two end points of $\Gamma$. We assume that $\arg \left(x_{0}\right) \geq \arg \left(x_{1}\right)$. Note that $x_{0}=x_{1}$ only if $\Gamma$ is a singleton, as we assume that $\Omega$ is not a disk centered at the origin.

Let $s$ be a signed arc length parameter for $\partial \Omega$ with a negative sign in the counter-clockwise direction starting at $x_{0}$. Then, $\partial \Omega$ and $\partial B$ can be represented as parametric functions $\Phi_{\Omega}$ and $\Phi_{B}$, respectively, near $\Gamma$, such that for some $s_{1} \geq 0$

$$
\begin{aligned}
& x_{0}=\Phi_{\Omega}(0)=\Phi_{B}(0), \\
& x_{1}=\Phi_{\Omega}\left(s_{1}\right)=\Phi_{B}\left(s_{1}\right) .
\end{aligned}
$$

Additionally, we define a distance function $\phi$,

$$
\phi(s)=\left|\Phi_{B}(s)-\Phi_{\Omega}(s)\right|, \quad-\delta \leq s \leq s_{1}+\delta .
$$

Clearly, $\phi(s)=0$ for $0 \leq s \leq s_{1}$, and $\phi$ decreases in $(-\delta, 0)$ and increases in $\left(s_{1}, s_{1}+\delta\right)$ by taking $\delta>0$ to be sufficiently small.

For a given $0<\varepsilon<\delta$, let $y_{0}=\Phi_{\Omega}(-\varepsilon)$ and $y_{1}=\Phi_{\Omega}\left(s_{1}+\varepsilon\right)$. Now, we rotate $\Omega$ about the origin clockwise when $\left|y_{0}\right| \geq\left|y_{1}\right|$ or counter-clockwise when $\left|y_{0}\right| \leq\left|y_{1}\right|$, so that $Q_{\varepsilon}\left(y_{i}\right)$ meets the arc $\widehat{x_{1-i} y_{1-i}}$ of $\partial \Omega\left(i=0\right.$ for $\left|y_{0}\right| \geq\left|y_{1}\right|$ and $i=1$ for $\left.\left|y_{0}\right| \leq\left|y_{1}\right|\right)$. Here, $Q_{\varepsilon}$ denotes the rotation operator depending on $\varepsilon$. Although using $Q_{ \pm \varepsilon}$ is more appropriate, by taking into account the rotation direction, we omit \pm . There is only one point $\eta_{i} \in \widehat{x_{1-i} y_{1-i}}(i=0,1)$ such that

$$
\eta_{i}=Q_{\varepsilon}\left(y_{i}\right)
$$

as $\phi(s)$ is a monotone in $(-\varepsilon, 0)$ and $\left(s_{1}, s_{1}+\varepsilon\right)$. We define the domain $D_{\varepsilon}$ bounded by three arcs,

$$
\begin{aligned}
\widehat{Q_{\varepsilon}\left(x_{i}\right) x_{1-i}} & \subset \partial B, \\
\widehat{x_{1-i} \eta_{i}} & \subset \partial \Omega \\
\widehat{Q_{\varepsilon}\left(y_{i}\right) Q_{\varepsilon}\left(x_{i}\right)} & \subset Q_{\varepsilon}(\partial \Omega) .
\end{aligned}
$$

By the construction of $D_{\varepsilon}$, we see that

$$
D_{\varepsilon} \subset \mathbb{R}^{2} \backslash\left(\bar{\Omega} \cup \overline{Q_{\varepsilon}(\Omega)}\right)
$$

and is enclosed in a circle with radius $\varepsilon$.

Now, we fix $d=d_{0}$ and $k=k_{0}$ and let $Q$ be any rotation operator. Then, Lemma 2 or (6) gives

$$
\begin{aligned}
v\left(x, d_{0}, k_{0}\right) & =v^{s}\left(x, d_{0}, k_{0}\right)+e^{i k x \cdot d_{0}} \\
& =u^{s}\left(Q^{-1}(x), Q^{-1}\left(d_{0}\right), k_{0}\right)+e^{i k x \cdot d_{0}} \quad \text { in } \mathbb{R}^{2} \backslash \bar{B} .
\end{aligned}
$$

Recall that $B$ is the smallest disk that contains $\Omega$. Moreover, the hypothesis of Theorem 1, (3), together with Lemma 3 yields

$$
u^{s}\left(Q^{-1}(x), Q^{-1}\left(d_{0}\right), k_{0}\right)=u^{s}\left(x, d_{0}, k_{0}\right) \quad \text { in } \mathbb{R}^{2} \backslash \bar{B}
$$

Thus, we have

$$
v^{s}\left(x, d_{0}, k_{0}\right)=u^{s}\left(x, d_{0}, k_{0}\right) \quad \text { in } \mathbb{R}^{2} \backslash \bar{B}
$$


and

$$
v\left(x, d_{0}, k_{0}\right)=u\left(x, d_{0}, k_{0}\right) \quad \text { in } \mathbb{R}^{2} \backslash \bar{B}
$$

By taking $Q=Q_{\varepsilon},(8)$ shows that $v$ and $u$ have the same Cauchy data on $\widehat{Q_{\varepsilon}\left(x_{i}\right) x_{1-i}} \subset \partial B$. As $v$ and $u$ solve (1a) in $D_{\varepsilon}$, the unique continuation principle gives

$$
v\left(x, d_{0}, k_{0}\right)=u\left(x, d_{0}, k_{0}\right) \quad \text { in } D_{\varepsilon},
$$

and thus

$$
u\left(x, d_{0}, k_{0}\right)=v\left(x, d_{0}, k_{0}\right)=0 \quad \text { on } \widehat{Q_{\varepsilon}\left(y_{i}\right) Q_{\varepsilon}\left(x_{i}\right)} \subset Q_{\varepsilon}(\partial \Omega) .
$$

Furthermore, we claim that

$$
u\left(x, d_{0}, k_{0}\right)=0 \quad \text { on } \widehat{Q_{\varepsilon}\left(x_{i}\right) x_{1-i}} \subset \partial B .
$$

Indeed, for $x \in \widehat{Q_{\varepsilon}\left(x_{i}\right) x_{1-i}} \subset \partial B$, there is $Q$ such that $Q\left(x_{i}\right)=x\left(i=0\right.$ for $\left|y_{0}\right| \geq\left|y_{1}\right|$ and $i=1$ for $\left.\left|y_{0}\right| \leq\left|y_{1}\right|\right)$. Then (7) implies

$$
u\left(x, d_{0}, k_{0}\right)=u\left(Q\left(x_{i}\right), d_{0}, k_{0}\right)=u\left(x_{i}, Q^{-1}\left(d_{0}\right), k_{0}\right)
$$

As $x_{i} \in \partial \Omega$ and $x$ is an arbitrary point in $\widehat{Q_{\varepsilon}\left(x_{i}\right) x_{1-i}}$, the boundary condition (10) is valid. Since $u\left(x, d_{0}, k_{0}\right)$ satisfies the zero Dirichlet boundary condition on $\partial \Omega$,

$$
u\left(x, d_{0}, k_{0}\right)=0 \quad \text { on } \widehat{x_{1-i} \eta_{i}} \subset \partial \Omega .
$$

Combining the boundary conditions $(9),(10)$, and (11) shows that $u\left(x, d_{0}, k_{0}\right)$ is a solution to the following Dirichlet boundary value problem,

$$
\begin{aligned}
& \Delta w+k_{0}^{2} w=0 \text { in } D_{\varepsilon}, \\
& w=0 \text { on } \partial D_{\varepsilon} .
\end{aligned}
$$

That is, $u\left(x, d_{0}, k_{0}\right)$ is an eigenfunction of the negative Laplacian in $D_{\varepsilon}$ under the Dirichlet boundary condition for any $\varepsilon>0$ with eigenvalue $k_{0}^{2}$. On the other hand, as $D_{\varepsilon}$ is a subset of the disk $B_{\varepsilon}$ with radius $\varepsilon$, the strong monotonicity property (see e.g. [12]) yields

$$
k_{0}^{2} \leq \lambda_{n}
$$

where $\lambda_{n}$ is the $n$th eigenvalue for $B_{\varepsilon}$. Now, we take $\varepsilon$ to be sufficiently small, so that there is no eigenvalue for $B_{\varepsilon}$. It follows that

$$
u\left(x, d_{0}, k_{0}\right) \equiv 0 \quad \text { in } D_{\varepsilon} .
$$

We use the unique continuation principle again to conclude that

$$
u\left(x, d_{0}, k_{0}\right) \equiv 0 \quad \text { in } \mathbb{R}^{2} \backslash \bar{\Omega}
$$

which yields a contradiction as $u_{\infty} \equiv 0$. Hence, we have proved Theorem 1.

\section{$3 \quad$ Further remarks}

We proved Theorem 1 based on the unique continuation principle and the monotonicity of eigenvalues of the negative Laplacian in two dimensions. This idea can be applied to the sound-hard case by a simple modification. It suffices to show that $u\left(x, d_{0}, k_{0}\right)$ has zero Neumann boundary condition on $\partial D_{\varepsilon}$. The same argument as that used in Section 2 gives that

$$
\frac{\partial}{\partial \nu} u\left(x, d_{0}, k_{0}\right)=0 \quad \text { on } \widehat{x_{1-i} \eta_{i}} \cup \widehat{Q_{\varepsilon}\left(y_{i}\right) Q_{\varepsilon}\left(x_{i}\right)}
$$

As $\partial \Omega$ is tangent to $\partial B$ at any point on $\Gamma$, the same argument used to derive the boundary condition (10) yields

$$
\frac{\partial}{\partial \nu} u\left(x, d_{0}, k_{0}\right)=0 \quad \text { on } \widehat{Q_{\varepsilon}\left(x_{i}\right) x_{1-i}}
$$


It is also known (see e.g. [13]) that the Neumann eigenvalues have monotonicity and the smallest positive eigenvalue $\mu_{1}$ satisfies

$$
\mu_{1} \geq \frac{\pi^{2}}{4 \varepsilon^{2}} .
$$

By taking sufficiently small $\varepsilon$, we can conclude that $u\left(x, d_{0}, k_{0}\right)=0$ in $D_{\varepsilon}$, which yields a contradiction as well. The proposed method may be applied in a three-dimensional space as well, by constructing $D_{\varepsilon}$ from two rotations.

The proposed method is new and is applicable to the other types of symmetric problems, because the main ingredients are the unique continuation principle and monotonicity of eigenvalues, which are general theory in analysis. In particular, we expect that this idea may be adapted to several types of inverse scattering problems with reduced data, such as phaseless data.

\section{References}

[1] David Colton and Rainer Kress. Inverse acoustic and electromagnetic scattering theory, volume 93 of Applied Mathematical Sciences. Springer, New York, third edition, 2013.

[2] Alexander G. Ramm. Scattering by obstacles, volume 21 of Mathematics and its Applications. D. Reidel Publishing Co., Dordrecht, 1986.

[3] David Colton and Rainer Kress. Looking back on inverse scattering theory. SIAM Rev., 60(4):779-807, 2018.

[4] Samuel N. Karp. Far field amplitudes and inverse diffraction theory. In Electromagnetic waves, pages 291-300. Univ. of Wisconsin Press, Madison, Wis., 1962.

[5] David Colton and Andreas Kirsch. Karp's theorem in acoustic scattering theory. Proc. Amer. Math. Soc., 103(3):783-788, 1988.

[6] David Colton and Rainer Kress. Karp's theorem in electromagnetic scattering theory. Proc. Amer. Math. Soc., 104(3):764-769, 1988.

[7] P. A. Martin and G. Dassios. Karp's theorem in elastodynamic inverse scattering. Inverse Problems, 9(1):97-111, 1993.

[8] Alexander G. Ramm. Symmetry properties of scattering amplitudes and applications to inverse problems. $J$. Math. Anal. Appl., 156(2):333-340, 1991.

[9] Alexander G. Ramm. Symmetry problem. Proc. Amer. Math. Soc., 141(2):515-521, 2013.

[10] Alexander G. Ramm. Symmetry problems for the Helmholtz equation. Appl. Math. Lett., 96:122-125, 2019.

[11] Fioralba Cakoni and David Colton. A Qualitative Approach to Inverse Scattering Theory. Applied Mathematical Sciences. Springer US, 2013.

[12] Rolf Leis. Initial boundary value problems in mathematical physics. Teubner, 1986.

[13] L. E. Payne and H. F. Weinberger. An optimal Poincaré inequality for convex domains. Arch. Rational Mech. Anal., 5:286-292 (1960), 1960. 